\title{
Special Matrices in Constructing Mutually Unbiased Maximally Entangled Bases in $C^{2} \otimes C^{4}$
}

\author{
Jun Zhang, Qiang Yang, Hua Nan, Yuanhong Tao* \\ Department of Mathematics, College of Sciences, Yanbian University, Yanji, China \\ Email: taoyuanhong12@126.com
}

Received 2 June 2015; accepted 20 June 2015; published 25 June 2015

Copyright (C) 2015 by authors and OALib.

This work is licensed under the Creative Commons Attribution International License (CC BY). http://creativecommons.org/licenses/by/4.0/
(c) (i)
Open Access

\section{Abstract}

Some special matrices can really help us to construct more than two mutually unbiased maximally entangled bases in $C^{2} \otimes C^{4}$. Through detailed analysis of the necessary and sufficient conditions of two maximally entangled bases to be mutually unbiased, we find these special matrices. Taking one such kind of matrix, we present the steps of constructing five mutually unbiased maximally entangled bases in $C^{2} \otimes C^{4}$.

\section{Keywords}

Maximally Entangled States, Mutually Unbiased Bases, Pauli Matrices

Subject Areas: Algebra, Quantum Mechanics, Theoretical Physics

\section{Introduction}

Mutually unbiased maximally entangled bases (MUMEBs) are an interesting topic combining mutually unbiased bases (MUBs) and maximally entangled states. Mutually unbiased bases play an central role in quantum kinematics [1], quantum state tomography [2]-[4] and many tasks in quantum information processing, such as quantum key distribution [5], cryptographic protocols [6] [7], mean king problem [8], quantum teleportation and superdense coding [9]-[11]. Maximally entangled state is central both to the foundations of quantum mechanics and to quantum information and computation [12]-[24].

A state $|\varphi\rangle$ is said to be a $d \otimes d^{\prime}\left(d^{\prime} \geq d\right)$ maximally entangled state if and only if for an arbitrary given

"Corresponding author.

How to cite this paper: Zhang, J., Yang, Q., Nan, H. and Tao, Y.H. (2015) Special Matrices in Constructing Mutually Unbiased Maximally Entangled Bases in $C^{2} \otimes C^{4}$. Open Access Library Journal, 2: e1620.

http://dx.doi.org/10.4236/oalib.1101620 
orthonormal complete basis $\left\{\left|i_{A}\right\rangle\right\}$ of subsystem $\boldsymbol{A}$, there exists an orthonormal basis $\left\{\left|i_{B}\right\rangle\right\}$ of subsystem $\boldsymbol{B}$ such that $|\varphi\rangle$ can be written as $|\varphi\rangle=\sum_{i=0}^{d-1}\left|i_{A}\right\rangle \otimes\left|i_{B}\right\rangle$ [24]. Two orthonormal bases $\boldsymbol{B}_{1}=\left\{\left|\phi_{i}\right\rangle\right\}_{i=1}^{d}$ and $\boldsymbol{B}_{2}=\left\{\left|\psi_{i}\right\rangle\right\}_{i=1}^{d}$ of $C^{d}$ are mutually unbiased if and only if $\left|\left\langle\varphi_{i} \mid \psi_{i}\right\rangle\right|=\frac{1}{\sqrt{d}}, \forall i, j=1,2, \cdots, d$. A set of orthonormal bases $\boldsymbol{B}_{1}, \boldsymbol{B}_{2}, \cdots, \boldsymbol{B}_{m}$ in $C^{d}$ are said to be a set of mutually unbiased bases if every pair of bases in the set is mutually unbiased.

Mutually unbiased bases are recently combined with other bases, such as product basis (PB) [25], unextendible product basis (UPB) [26], unextendible maximally entangled basis (UMEB) [27]-[32] and maximally entangled basis (MEB) [33]-[35]. The MEB is a set of orthonormally maximally entangled states in $C^{d} \otimes C^{d}$ consisting of $d^{2}$ vectors. In [33]-[35], by systematically constructing MEBs, the concrete construction of pairs of MUMEBs in bipartite systems $C^{d} \otimes C^{k d}\left(k \in \mathrm{Z}^{+}\right)$is studied.

In this note, we study the problem of constructing more than two mutually unbiased maximally entangled bases in bipartite spaces $C^{2} \otimes C^{4}$. Through the sufficient and necessary conditions of two maximally entangled bases to be mutually unbiased, we find the special matrices and present steps of using special matrix to construct five mutually unbiased maximally entangled bases in $C^{2} \otimes C^{4}$.

\section{Main Results}

We first recall the sufficient and necessary conditions of two maximally entangled bases to be mutually unbiased in $C^{2} \otimes C^{4}$.

Let $\{|0\rangle,|1\rangle\}$ be the orthonormal basis in $C^{2},\left(\left|e^{\prime}\right\rangle\right)=\left\{\left|0^{\prime}\right\rangle,\left|1^{\prime}\right\rangle,\left|2^{\prime}\right\rangle,\left|3^{\prime}\right\rangle\right\}$ and $\left(\left|a^{\prime}\right\rangle\right)=\left\{\left|a_{0}^{\prime}\right\rangle,\left|a_{1}^{\prime}\right\rangle,\left|a_{2}^{\prime}\right\rangle,\left|a_{3}^{\prime}\right\rangle\right\}$ be two othonormal bases in $C^{4}, \boldsymbol{A}$ denotes the transition matrix between them, that is $\left(\left|a^{\prime}\right\rangle\right)=A\left(\left|e^{\prime}\right\rangle\right)$, i.e., $\left(\left|a_{i}^{\prime}\right\rangle\right)=\sum_{j=0}^{3} a_{i j}\left(\left|j^{\prime}\right\rangle\right), a_{i j}$ are entries of the matrix $\boldsymbol{A}$.

We first consider two MEBs in $C^{2} \otimes C^{4}[33]$ as follows:

$$
\begin{aligned}
& \left|\phi_{i}^{j}\right\rangle=\frac{1}{\sqrt{2}}\left(\sigma_{i} \otimes I_{4}\right)\left(|0\rangle\left|2 j^{\prime}\right\rangle+|1\rangle\left|2 j+1^{\prime}\right\rangle\right), \quad i=0,1,2,3 ; j=0,1 . \\
& \left|\psi_{i}^{j}\right\rangle=\frac{1}{\sqrt{2}}\left(\sigma_{i} \otimes I_{4}\right)\left(|0\rangle\left|a_{2 j}^{\prime}\right\rangle+|1\rangle\left|a_{2 j+1}^{\prime}\right\rangle\right), \quad i=0,1,2,3 ; j=0,1 .
\end{aligned}
$$

where $\sigma_{i}, i=1,2,3$ are Pauli matrices and $\sigma_{0}=I_{2}$.

From [33], the above two MEBs (1) and (2) in $C^{2} \otimes C^{4}$ are mutually unbiased if and only if the matrices $\boldsymbol{A}$ satisfy the following relations:

$$
\left|\sum_{p=0}^{1}(-1)^{\ell p} A_{p+2 j, p \oplus q+2 i}\right|=\frac{1}{\sqrt{2}}
$$

where $i, j=0,1 ; \ell, q=0,1 ;$ and $p \oplus q$ denotes $p+q \bmod 2$.

To visualize the conditions (3), we divide the transition matrix $\boldsymbol{A}$ into 4 submatrices of $2 \times 2$ from left to right, then the conditions (3) hold if and only if each $2 \times 2$ submatrix satisfying the similar conditions as follows (we might take the upper left submatrix as a representative):

$$
\begin{aligned}
& \left|a_{11}+a_{22}\right|=\left|a_{11}-a_{22}\right|=\frac{1}{\sqrt{2}} ; \\
& \left|a_{12}+a_{21}\right|=\left|a_{12}-a_{21}\right|=\frac{1}{\sqrt{2}} ;
\end{aligned}
$$

From [33], it is easy to find matrices satisfying the above conditions (4) such as 


$$
\frac{1}{2}\left(\begin{array}{cccc}
-i & -1 & 1 & i \\
-i & -1 & -1 & i \\
-i & 1 & -1 & -i \\
-i & 1 & 1 & i
\end{array}\right) ; \frac{1}{2}\left(\begin{array}{cccc}
1 & 1 & -i & -i \\
i & i & -1 & -1 \\
i & -i & -1 & 1 \\
1 & -1 & -i & i
\end{array}\right) ; \frac{1}{2}\left(\begin{array}{cccc}
-i & -i & -i & -i \\
1 & 1 & -1 & -1 \\
-1 & 1 & 1 & -1 \\
-i & i & -i & i
\end{array}\right) ; \frac{1}{2}\left(\begin{array}{cccc}
1 & 1 & 1 & 1 \\
i & i & -i & -i \\
i & -i & i & -i \\
1 & -1 & -1 & 1
\end{array}\right)
$$

In this note, we want to find more than two MUMEBs, so how to find the third MEB mutually unbiased with the above two MEBs (1) and (2), it depends on the property transit matrix satisfied. Suppose that $\left(\left|b^{\prime}\right\rangle\right)=\left\{\left|b_{0}^{\prime}\right\rangle,\left|b_{1}^{\prime}\right\rangle,\left|b_{2}^{\prime}\right\rangle,\left|b_{3}^{\prime}\right\rangle\right\}$ be the third orthonormal basis in $C^{4}$, and $\boldsymbol{B}$ denotes the transition matrix between $\left(\left|b^{\prime}\right\rangle\right)$ and $\left(\left|a^{\prime}\right\rangle\right)$, that is $\left(\left|b^{\prime}\right\rangle\right)=B\left(\left|a^{\prime}\right\rangle\right)$, i.e. $\left(\left|b_{i}^{\prime}\right\rangle\right)=\sum_{j=0}^{3} b_{i j}\left(\left|a_{j}^{\prime}\right\rangle\right), b_{i j}$ are entries of the matrix $\boldsymbol{B}$. Then according to [33], we have the third MEB as follows

$$
\left|\lambda_{i}^{j}\right\rangle=\frac{1}{\sqrt{2}}\left(\sigma_{i} \otimes I_{4}\right)\left(|0\rangle\left|b_{2 j}^{\prime}\right\rangle+|1\rangle\left|b_{2 j+1}^{\prime}\right\rangle\right), \quad i=0,1,2,3 ; j=0,1
$$

Then, the above three MEBs in $C^{2} \otimes C^{4}$ are mutually unbiased if and only if the matrices $\boldsymbol{A}, \boldsymbol{B}$ and $\boldsymbol{B} \boldsymbol{A}$ all satisfy the conditions (4) simultaneously.

Since the transit matrix $\boldsymbol{A}$ is easy to choose, we really want to know the way to construct matrix $B$. Assume that

$$
\boldsymbol{B}=\boldsymbol{A P}
$$

where $\boldsymbol{P}$ is a $2 \times 2$ matrix, if $\boldsymbol{A}$ is known, how can we choose the matrix $\boldsymbol{P}$ to assure $\boldsymbol{B}$ and $\boldsymbol{B} \boldsymbol{A}$ all satisfy the conditions (4)? For simplicity, we can first assume that $\boldsymbol{P}$ be a diagonal block matrix

$$
\boldsymbol{P}=\left(\begin{array}{cccc}
p_{11} & p_{12} & 0 & 0 \\
p_{21} & p_{22} & 0 & 0 \\
0 & 0 & p_{33} & p_{34} \\
0 & 0 & p_{43} & p_{44}
\end{array}\right)
$$

then we have

$$
\left(\begin{array}{ll}
b_{11} & b_{12} \\
b_{21} & b_{22}
\end{array}\right)=\left(\begin{array}{ll}
a_{11} & a_{12} \\
a_{21} & a_{22}
\end{array}\right) \cdot\left(\begin{array}{ll}
p_{11} & p_{12} \\
p_{21} & p_{22}
\end{array}\right)=\left(\begin{array}{ll}
a_{11} p_{11}+a_{12} p_{21} & a_{11} p_{12}+a_{12} p_{22} \\
a_{21} p_{11}+a_{22} p_{21} & a_{21} p_{12}+a_{22} p_{22}
\end{array}\right)
$$

Since $\boldsymbol{B}$ satisfy the conditions (4), then we have

$$
\begin{aligned}
& \left|a_{11} p_{11}+a_{12} p_{21}+a_{21} p_{12}+a_{22} p_{22}\right|=\left|a_{11} p_{11}+a_{12} p_{21}-a_{21} p_{12}-a_{22} p_{22}\right|=\frac{1}{\sqrt{2}} \\
& \left|a_{11} p_{12}+a_{12} p_{22}+a_{21} p_{11}+a_{22} p_{21}\right|=\left|a_{11} p_{12}+a_{12} p_{22}-a_{21} p_{11}-a_{22} p_{21}\right|=\frac{1}{\sqrt{2}}
\end{aligned}
$$

thus we must have

$$
p_{11}=p_{22}=0 \text { or } p_{12}=p_{21}=0 .
$$

It follows from the unitarity of matrix $\boldsymbol{P}$ that

$$
\left\{\begin{array} { l } 
{ p _ { 1 1 } = p _ { 2 2 } = 0 ; } \\
{ p _ { 1 2 } { } ^ { 2 } = p _ { 2 1 } ^ { 2 } = \pm 1 ; }
\end{array} \text { or } \left\{\begin{array}{l}
p_{12}=p_{21}=0 \\
p_{11}^{2}=p_{22}^{2}= \pm 1
\end{array}\right.\right.
$$

Similarly, we can have

$$
\left\{\begin{array} { l } 
{ p _ { 3 3 } = p _ { 4 4 } = 0 ; } \\
{ p _ { 3 4 } { } ^ { 2 } = p _ { 4 3 } { } ^ { 2 } = \pm 1 ; }
\end{array} \text { or } \left\{\begin{array}{l}
p_{34}=p_{43}=0 \\
p_{33}{ }^{2}=p_{44}{ }^{2}= \pm 1
\end{array}\right.\right.
$$

so there are many choices about the values of $p_{11}, p_{22}, p_{12}, p_{21}, p_{33}, p_{34}, p_{43}, p_{44}$. To avoid the trivial diagonal case of matrix $\boldsymbol{P}$, we may take $p_{12}=p_{21}=p_{33}=p_{44}=0$, then the values of $p_{11}, p_{22}, p_{43}, p_{44}$ can be divided 
into the following two cases:

$$
\text { Case I: }\left\{\begin{array} { l } 
{ p _ { 1 1 } { } ^ { 2 } = p _ { 2 2 } { } ^ { 2 } = - 1 ; } \\
{ p _ { 3 4 } { } ^ { 2 } = p _ { 4 3 } { } ^ { 2 } = 1 ; }
\end{array} \text { Case II: } \left\{\begin{array}{l}
p_{11}{ }^{2}=p_{22}{ }^{2}=1 ; \\
p_{34}{ }^{2}=p_{43}{ }^{2}=-1 ;
\end{array}\right.\right.
$$

We first discuss the case I. Obviously, there are many forms of $\boldsymbol{P}$ satisfying the above property, such as

$$
\left(\begin{array}{cccc}
i & 0 & 0 & 0 \\
0 & i & 0 & 0 \\
0 & 0 & 0 & 1 \\
0 & 0 & 1 & 0
\end{array}\right) ;\left(\begin{array}{cccc}
i & 0 & 0 & 0 \\
0 & -i & 0 & 0 \\
0 & 0 & 0 & -1 \\
0 & 0 & 1 & 0
\end{array}\right) ; \quad\left(\begin{array}{cccc}
-i & 0 & 0 & 0 \\
0 & i & 0 & 0 \\
0 & 0 & 0 & -1 \\
0 & 0 & -1 & 0
\end{array}\right) ;\left(\begin{array}{cccc}
-i & 0 & 0 & 0 \\
0 & -i & 0 & 0 \\
0 & 0 & 0 & 1 \\
0 & 0 & 1 & 0
\end{array}\right) ;
$$

No loss of generality, we first choose

$$
\boldsymbol{A}=\frac{1}{2}\left(\begin{array}{cccc}
1 & 1 & 1 & 1 \\
i & i & -i & -i \\
i & -i & i & -i \\
1 & -1 & -1 & 1
\end{array}\right) ; \quad \boldsymbol{P}=\left(\begin{array}{cccc}
i & 0 & 0 & 0 \\
0 & i & 0 & 0 \\
0 & 0 & 0 & 1 \\
0 & 0 & 1 & 0
\end{array}\right)
$$

then we have

$$
\boldsymbol{B}=\boldsymbol{A} \boldsymbol{P}=\frac{1}{2}\left(\begin{array}{cccc}
i & i & 1 & 1 \\
-1 & -1 & -i & -i \\
-1 & 1 & -i & i \\
i & -i & 1 & -1
\end{array}\right) ; \quad \boldsymbol{B} \boldsymbol{A}=\frac{1}{2}\left(\begin{array}{cccc}
i & -1 & i & 1 \\
-i & -1 & i & -1 \\
i & -1 & -i & -1 \\
i & 1 & i & -1
\end{array}\right)
$$

It is direct to verify that the transformation matrix $\boldsymbol{B}$ and $\boldsymbol{B} \boldsymbol{A}$ both satisfy the conditions (4), then the MEBs (1), (2) and (5) in $C^{2} \otimes C^{4}$ are mutually unbiased.

Let $\boldsymbol{A}_{1}=\boldsymbol{B} \boldsymbol{A}$, then

$$
\boldsymbol{C}=\boldsymbol{A}_{1} \boldsymbol{P}=\frac{1}{2}\left(\begin{array}{cccc}
-1 & -i & 1 & i \\
1 & -i & -1 & i \\
-1 & -i & -1 & -i \\
-1 & i & -1 & i
\end{array}\right)
$$

Denoting $\left(\left|c^{\prime}\right\rangle\right)=\left\{\left|c_{0}^{\prime}\right\rangle,\left|c_{1}^{\prime}\right\rangle,\left|c_{2}^{\prime}\right\rangle,\left|c_{3}^{\prime}\right\rangle\right\}$ be the fourth orthonormal basis in $C^{4}$, and $\boldsymbol{C}$ denotes the transition matrix between $\left(\left|c^{\prime}\right\rangle\right)$ and $\left(\left|b^{\prime}\right\rangle\right)$, that is $\left(\left|c^{\prime}\right\rangle\right)=\boldsymbol{C}\left(\left|b^{\prime}\right\rangle\right)$, then the fourth MEB in $C^{2} \otimes C^{4}$ can be constructed as follows:

$$
\left|\mu_{i}^{j}\right\rangle=\frac{1}{\sqrt{2}}\left(\sigma_{i} \otimes I_{4}\right)\left(|0\rangle\left|c_{2 j}^{\prime}\right\rangle+|1\rangle\left|c_{2 j+1}^{\prime}\right\rangle\right), \quad i=0,1,2,3 ; j=0,1 .
$$

Obviously, $\left(\left|c^{\prime}\right\rangle\right)=\boldsymbol{C} \boldsymbol{A}_{1}\left(\left|e^{\prime}\right\rangle\right),\left(\left|c^{\prime}\right\rangle\right)=\boldsymbol{C B}\left(\left|a^{\prime}\right\rangle\right)$ and

$$
\boldsymbol{C A}_{1}=\frac{1}{2}\left(\begin{array}{cccc}
-1 & i & -i & -1 \\
-1 & i & i & 1 \\
-i & 1 & 1 & i \\
-i & 1 & -1 & -i
\end{array}\right) ; \quad \boldsymbol{C B}=\frac{1}{2}\left(\begin{array}{cccc}
-1 & 1 & -1 & -1 \\
i & i & i & -i \\
1 & -1 & -1 & -1 \\
-i & -i & i & -i
\end{array}\right)
$$

It is easy to check the above matrices $\boldsymbol{C}, \boldsymbol{C} \boldsymbol{A}_{1}$ and $\boldsymbol{C B}$ all satisfy the conditions (4), so the fourth MEB (13) is mutually unbiased with the former three bases (1), (2) and (5) in $C^{2} \otimes C^{4}$.

Moreover, let $\boldsymbol{A}_{2}=\boldsymbol{C} \boldsymbol{A}_{1}$, then

$$
\boldsymbol{D}=\boldsymbol{A}_{2} \boldsymbol{P}=\frac{1}{2}\left(\begin{array}{cccc}
-i & -1 & -1 & -i \\
-i & -1 & 1 & i \\
1 & i & i & 1 \\
1 & i & -i & -1
\end{array}\right)
$$


Denoting $\left(\left|d^{\prime}\right\rangle\right)=\left\{\left|d_{0}^{\prime}\right\rangle,\left|d_{1}^{\prime}\right\rangle,\left|d_{2}^{\prime}\right\rangle,\left|d_{3}^{\prime}\right\rangle\right\}$ be the fifth orthonormal basis in $C^{4}$, and $\boldsymbol{D}$ denotes the transition matrix between $\left(\left|d^{\prime}\right\rangle\right)$ and $\left(\left|c^{\prime}\right\rangle\right)$, that is $\left(\left|d^{\prime}\right\rangle\right)=\boldsymbol{D}\left(\left|c^{\prime}\right\rangle\right)$, then the fourth MEB in $C^{2} \otimes C^{4}$ can be constructed as follows:

$$
\left|v_{i}^{j}\right\rangle=\frac{1}{\sqrt{2}}\left(\sigma_{i} \otimes I_{4}\right)\left(|0\rangle\left|d_{2 j}^{\prime}\right\rangle+|1\rangle\left|d_{2 j+1}^{\prime}\right\rangle\right), \quad i=0,1,2,3 ; j=0,1 .
$$

Obviously, $\left(\left|d^{\prime}\right\rangle\right)=\boldsymbol{D} \boldsymbol{A}_{2}\left(\left|e^{\prime}\right\rangle\right),\left(\left|d^{\prime}\right\rangle\right)=\boldsymbol{D C} \boldsymbol{B}\left(\left|a^{\prime}\right\rangle\right),\left(\left|d^{\prime}\right\rangle\right)=\boldsymbol{D} \boldsymbol{C}\left(\left|b^{\prime}\right\rangle\right)$ and

$$
\boldsymbol{D} \boldsymbol{A}_{2}=\frac{1}{2}\left(\begin{array}{cccc}
i & -i & -1 & -1 \\
1 & 1 & -i & i \\
-i & i & -1 & -1 \\
-1 & -1 & -i & i
\end{array}\right) ; \quad \boldsymbol{D C} \boldsymbol{B}=\frac{1}{2}\left(\begin{array}{cccc}
-1 & -i & 1 & i \\
1 & -i & -1 & i \\
-1 & -i & -1 & -i \\
-1 & i & -1 & i
\end{array}\right) ; \quad \boldsymbol{D C}=\frac{1}{2}\left(\begin{array}{cccc}
i & i & 1 & 1 \\
-1 & -1 & -i & -i \\
-1 & 1 & -i & i \\
i & -i & 1 & -1
\end{array}\right)
$$

One can directly check that the above matrices $\boldsymbol{D}, \boldsymbol{D} \boldsymbol{A}_{2}, \boldsymbol{D C B}$ and $\boldsymbol{D C}$ all satisfy the conditions (4), so the fifth MEB (14) is mutually unbiased with the former four bases (1), (2), (5) and (13) in $C^{2} \otimes C^{4}$.

Furthermore, let $\boldsymbol{A}_{3}=\boldsymbol{D} \boldsymbol{A}_{2}$, then

$$
\boldsymbol{F}=\boldsymbol{A}_{3} \boldsymbol{P}=\frac{1}{2}\left(\begin{array}{cccc}
-1 & 1 & -1 & -1 \\
i & i & i & -i \\
1 & -1 & -1 & -1 \\
-i & -i & i & -i
\end{array}\right)
$$

Denoting $\left(\left|f^{\prime}\right\rangle\right)=\left\{\left|f_{0}^{\prime}\right\rangle,\left|f_{1}^{\prime}\right\rangle,\left|f_{2}^{\prime}\right\rangle,\left|f_{3}\right\rangle\right\}$ be the fifth orthonormal basis in $C^{4}$, and $\boldsymbol{F}$ be the transition matrix between $\left(\left|f^{\prime}\right\rangle\right)$ and $\left(\left|d^{\prime}\right\rangle\right)$, that is $\left(\left|f^{\prime}\right\rangle\right)=\boldsymbol{F}\left(\left|d^{\prime}\right\rangle\right)$, then $\left(\left|f^{\prime}\right\rangle\right)=\boldsymbol{F} \boldsymbol{A}_{3}\left(\left|e^{\prime}\right\rangle\right)$ and

$$
\boldsymbol{F} A_{3}=\frac{1}{2}\left(\begin{array}{cccc}
1 & 1 & 1 & 1 \\
i & i & -i & -i \\
i & -i & i & -i \\
1 & -1 & -1 & 1
\end{array}\right)
$$

Since $\boldsymbol{F A}_{3}$ is exactly equal to $\boldsymbol{A}$, the sixth orthonormal basis $\left(\left|f^{\prime}\right\rangle\right)$ is equal to $\left(\left|a^{\prime}\right\rangle\right)$, thus using matrix $\boldsymbol{P}$, we can only get five MUMEBs (1), (2), (5), (13), (14) and no the sixth one.

Next, we discuss Case II of $p_{12}=p_{21}=p_{33}=p_{44}=0$. Now there are many forms of $\boldsymbol{P}$ satisfying the property, such as

$$
\left(\begin{array}{cccc}
1 & 0 & 0 & 0 \\
0 & 1 & 0 & 0 \\
0 & 0 & 0 & -i \\
0 & 0 & -i & 0
\end{array}\right) ;\left(\begin{array}{cccc}
1 & 0 & 0 & 0 \\
0 & -1 & 0 & 0 \\
0 & 0 & 0 & -i \\
0 & 0 & i & 0
\end{array}\right) ; \quad\left(\begin{array}{cccc}
-1 & 0 & 0 & 0 \\
0 & 1 & 0 & 0 \\
0 & 0 & 0 & i \\
0 & 0 & i & 0
\end{array}\right) ; \quad\left(\begin{array}{cccc}
-1 & 0 & 0 & 0 \\
0 & -1 & 0 & 0 \\
0 & 0 & 0 & i \\
0 & 0 & i & 0
\end{array}\right) ;
$$

If we take the same $\boldsymbol{A}$ in (12) and choose the following form of $\boldsymbol{P}$ :

$$
\boldsymbol{P}=\left(\begin{array}{cccc}
1 & 0 & 0 & 0 \\
0 & 1 & 0 & 0 \\
0 & 0 & 0 & -i \\
0 & 0 & -i & 0
\end{array}\right) ;
$$

similar to the above analysis, we can get the five MUMEBs in $C^{2} \otimes C^{4}$ in [33].

\section{Conclusion}

In this note, we have constructed five mutually unbiased maximally entangled bases in bipartite spaces $C^{2} \otimes C^{4}$ using special matrices. Thus, we have presented a method to construct more than two mutually un- 
biased maximally entangled bases in $C^{2} \otimes C^{4}$. Similar problems can be discussed in arbitrary bipartite spaces $C^{d} \otimes C^{k d}\left(k \in \mathrm{Z}^{+}\right)$.

\section{References}

[1] Durt, T., Englert, B.-G., Bengtsson, I. and Zyczkowski, K. (2010) On Mutually Unbiased Bases. International Journal of Quantum Information, 8, 535. http://dx.doi.org/10.1142/S0219749910006502

[2] Ivanovic, I.D. (1981) Geometrical Description of Quantal State Determination. Journal of Physics A: Mathematical and General, 14, 3241. http://dx.doi.org/10.1088/0305-4470/14/12/019

[3] Wootters, W.K. and Fields, B.D. (1989) Optimal State-Determination by Mutually Unbiased Measurements. Annals of Physics, 191, 363-381. http://dx.doi.org/10.1016/0003-4916(89)90322-9

[4] Englert, B.-G., Kaszlikowski, D., Kwek, L.C. and Chee, W.H. (2008) Wave-Particle Duality in Multi-Path Interferometers: General Concepts and Three-Path Interferometers. International Journal of Quantum Information, 6, 129-157.

[5] Cerf, N.J., Bourennane, M., Karlsson, A. and Gisin, N. (2002) Security of Quantum Key Distribution Using $d$-Level Systems. Physical Review Letters, 88, Article ID: 127902. http://dx.doi.org/10.1103/PhysRevLett.88.127902

[6] Xiong, Z.-X., Shi, H.-D., Wang, Y.-N., Jing, L., Lei, J., Mu, L.-Z. and Fan, H. (2012) General Quantum Key Distribution in Higher Dimension. Physics Review A, 85, Article ID: 012334.

[7] Brierley, S. (2009) Quanturn Key Distribution Highly Sensitive to Eavesdropping. arXiv: 0910.2578.

[8] Aharonov, Y. and Englert, B.G. (2001) The Mean King’s Problem: Spin 1. Zeitschrift Fur Naturforsch, 56a, 16.

[9] Durt, T. (2004) e-pnnt arXiv: quant-ph/0401046.

[10] Klimov, A.B., Sych, D., Sanchez-Soto, L.L. and Leuchs, G. (2009) Mutually Unbiased Bases and Generalized Bell States. Physical Review A, 79, Article ID: 052101. http://dx.doi.org/10.1103/PhysRevA.79.052101

[11] Revzen, M. (2010) Maximally Entangled States via Mutual Unbiased Collective Bases. Physical Review A, 81, Article ID: 012113. http://dx.doi.org/10.1103/PhysRevA.81.012113

[12] Vedral, V. (2002) The Role of Relative Entropy in Quantum Information Theory. Reviews of Modern Physics, 74, 197234. http://dx.doi.org/10.1103/RevModPhys.74.197

[13] Plenio, M.B. and Virmani, S. (2007) An Introduction to Entanglement Measures. Quantum Information and Computation, 7, 1-51.

[14] Horodecki, R., Horodecki, P., Horodecki, M. and Horodecki, K. (2009) Quantum Entanglement. Reviews of Modern Physics, 81, 865-942. http://dx.doi.org/10.1103/RevModPhys.81.865

[15] Benhelm, J., Kirchmair, G., Roos, C.F. and Blatt, R. (2008) Towards Fault-Tolerant Quantum Computing with Trapped Ions. Nature Physics, 4, 463-468. http://dx.doi.org/10.1038/nphys961

[16] Bennett, C.H., Brassard, G., Crepeau, C., Jozsa, R., Peres, A. and Wootters, W.K. (1993) Teleporting an Unknown Quantum State via Dual Classical and Einstein-Podolsky-Rosen Channels. Physical Review Letters, 70, 1895-1899. http://dx.doi.org/10.1103/PhysRevLett.70.1895

[17] Zhang, Q., Goebel, A., Wagenknecht, C., Chen, Y.A., Zhao, B., Yang, T., Mair, A., Schmied-Mayer, J. and Pan, J.W. (2006) Experimental Quantum Teleportation of a Two-Qubit Composite System. Nature Physics, 2, 678-682. http://dx.doi.org/10.1038/nphys417

[18] Modlawska, J. and Grudka, A. (2008) Non-Maximally Entangled States Can Be Better for Multiple Linear Optical Teleportation. Physical Review Letters, 100, Article ID: 110503. http://dx.doi.org/10.1103/PhysRevLett.100.110503

[19] Ishizaka, S. and Hiroshima, T. (2009) Quantum Teleportation Scheme by Selecting One of Multiple Output Ports. Physical Review A, 79, Article ID: 042306. http://dx.doi.org/10.1103/PhysRevA.79.042306

[20] Noh, C., Chia, A., Nha, H., Collett, M.J. and Carmichael, H.J. (2009) Quantum Teleportation of the Temporal Fluctuations of Light. Physical Review Letters, 102, Article ID: 230501. http://dx.doi.org/10.1103/PhysRevLett.102.230501

[21] Bennett, C.H. and Wiesner, S.J. (1992) Communication via One- and Two-Particle Operators on Einstein-PodolskyRosen States. Physical Review Letters, 69, 2881-2884. http://dx.doi.org/10.1103/PhysRevLett.69.2881

[22] Barreiro, J.T., Wei, T.C. and Kwiat, P.G. (2008) Beating the Channel Capacity Limit for Linear Photonic Superdense Coding. Nature Physics, 4, 282-286. http://dx.doi.org/10.1038/nphys919

[23] Bennett, C.H. and Di Vincenzo, D.P. (2000) Quantum Information and Computation. Nature, 404, $247-255$. http://dx.doi.org/10.1038/35005001

[24] Li, Z.G., Zhao, M.J., Fei, S.M., Fan, H. and Liu, W.M. (2012) Mixed Maximally Entangled States. Quantum Information \& Computation, 12, 63-73.

[25] McNulty, D. and Weigert, S. (2012) The Limited Role of Mutually Unbiased Product Bases in Dimension 6. Journal of 
Physics A: Mathematical and Theoretical, 45, Article ID: 102001. http://dx.doi.org/10.1088/1751-8113/45/10/102001

[26] Bennett, C.H., Divincenzo, D.P., Mor, T., Shor, P.W., Smolin, J.A. and Terhal, B.M. (1999) Unextendible Product Bases and Bound Entanglement. Physical Review Letters, 82, 5385-5388. http://dx.doi.org/10.1103/PhysRevLett.82.5385

[27] Bravyi, S. and Smolin, J.A. (2011) Unextendible Maximally Entangled Bases. Physical Review A, 84, Article ID: 042306. http://dx.doi.org/10.1103/PhysRevA.84.042306

[28] Chen, B. and Fei, S.M. (2013) Unextendible Maximally Entangled Bases and Mutually Unbiased Bases. Physical Review A, 88, Article ID: 034301. http://dx.doi.org/10.1103/PhysRevA.88.034301

[29] Nan, H., Tao, Y.H., Li, L.S. and Zhang, J. (2014) Unextendible Maximally Entangled Bases and Mutually Unbiased Bases in $\mathbb{C}^{d} \otimes \mathbb{C}^{d^{\prime}}$. International Journal of Theoretical Physics, 54, 927-932. http://dx.doi.org/10.1007/s10773-014-2288-1

[30] Li, M.S., Wang, Y.L. and Zheng, Z.J. (2014) Unextendible Maximally Entangled Bases in $\mathbb{C}^{d} \otimes \mathbb{C}^{d^{\prime}}$. Physical Review A, 89, Article ID: 062313. http://dx.doi.org/10.1103/PhysRevA.89.062313

[31] Wang, Y.L., Li, M.S. and Fei, S.M. (2014) Unextendible Maximally Entangled Bases in $\mathbb{C}^{d} \otimes \mathbb{C}^{d}$. Physical Review A, 90, Article ID: 034301. http://dx.doi.org/10.1103/PhysRevA.90.034301

[32] Nizamidin, H., Ma, T. and Fei, S.M. (2014) A Note on Mutually Unbiased Unextendible Maximally Entangled Bases in $\mathrm{C}^{2} \otimes \mathrm{C}^{3}$. International Journal of Theoretical Physics, 54, 326-333. http://dx.doi.org/10.1007/s10773-014-2227-1

[33] Tao, Y.H., Nan, H., Zhang, J. and Fei, S.M. (2015) Mutually Unbiased Maximally Entangled Bases in $C^{d} \otimes C^{k d}$. Quantum Information Processing, 14, 2291-2300. http://dx.doi.org/10.1007/s11128-015-0980-6

[34] Zhang, J., Tao, Y.H., Nan, H. and Fei, S.M. (2015) Construction of Mutually Unbiased Bases in $C^{d} \otimes C^{2 l d^{\prime}} . Q^{\text {Quntum }}$ Information Processing, 14, 2635-2644. http://dx.doi.org/10.1007/s11128-015-0961-9

[35] Tao, Y.H., Yang, Q., Zhang, J., Nan, H. and Li, L.S. (2015) The Concrete Construction of Mutually Unbiased Maximally Entangled Bases in $C^{d} \otimes C^{k d}$. Scientia Sinica Physica, Mechanica \& Astronomica, 45, Article ID: 060302. http://dx.doi.org/10.1360/SSPMA2015-00056 